\section{Cortical Linear Lesions in Wernicke's Encephalopathy: Can Diffusion-Weighted Imaging Herald Prognostic Information?}

To the Editor: Typical clinical features of Wernicke's encephalopathy are caused by vitamin $B_{1}$ deficiency and include confusion, ataxia, and ophthalmoplegia. ${ }^{1,2}$ Although it can occur in all malnourished patients, it is far more frequent in alcoholics. ${ }^{1,2}$ Brain MRI is a central diagnostic tool that usually discloses high signal lesions in T2-weighted imaging symmetrically affecting mammillary bodies, the thalamus, hypothalamus, periaqueductal gray matter, midbrain, and the cerebellum. ${ }^{2}$ Cortical damage and diffusion-weighted imaging findings were both seldom reported in Wernicke's encephalopathy.

\section{Case Report}

A 36-year-old man presented at the emergency department with an acute confusional state. He had excessive chronic daily alcohol abuse, averaging 200 grams per day, and he almost stopped eating in the prior week. Besides this, his prior medical history was unremarkable. When observed, he was confused, hallucinating, and disoriented; complete ophthalmoplegia, with multidirectional gaze-evoked nystagmus, and bilateral ataxia were seen, with no abnormality on motor or sensory testing. Brain MRI disclosed symmetrical high signal lesions in the mammillary bodies, in T2-weighted imaging FLAIR (Figure 1, panel A) and dif-

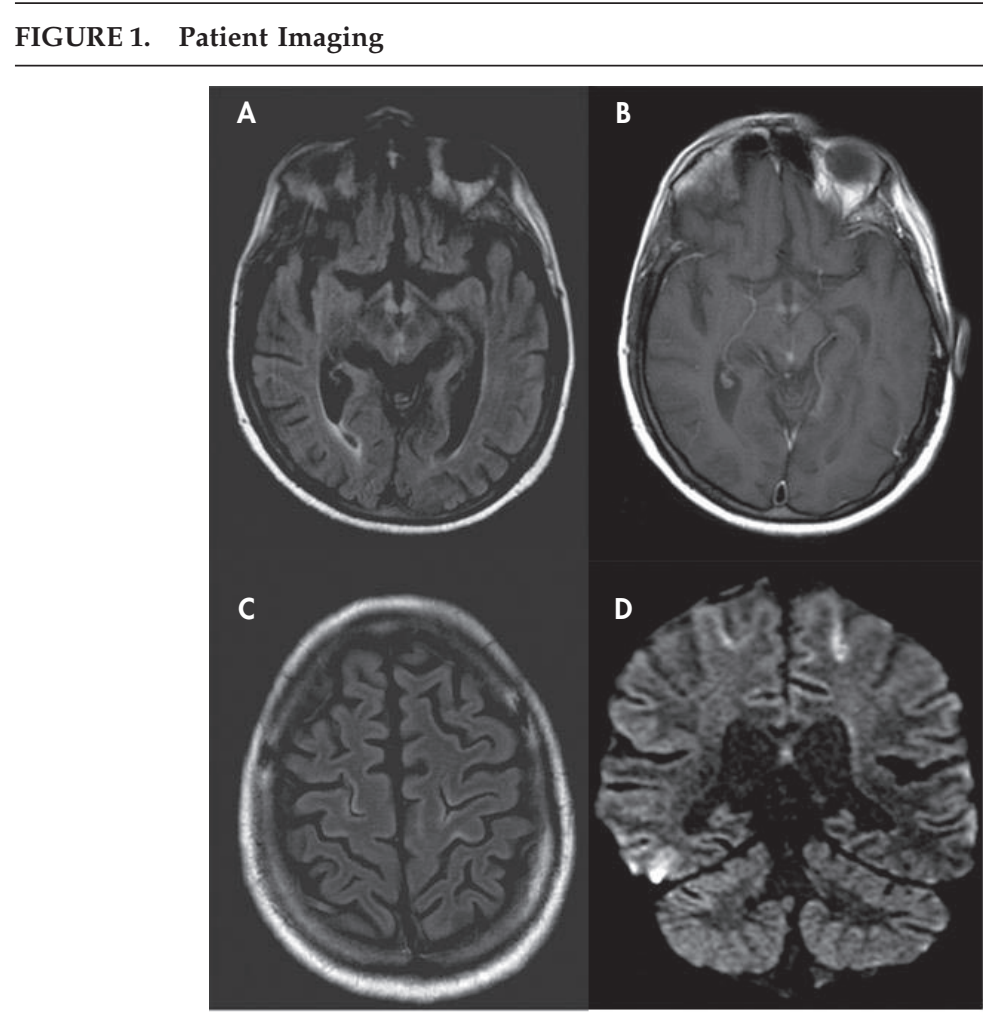

A: Axial FLAIR shows bilateral symmetrical hyperintensities in mammillary bodies, with slight enlargement; B: Gadolinium-enhanced T1-weighted image discloses blood-brain barrier disruption in the same location; C: Axial FLAIR shows bilateral cortical hyperintensities depicting precentral and central sulci; D: Coronal DWI reveals high signal lesions in the same location.

fusion weighted imaging, which was enhanced after gadolinium administration (Figure 1, panel B). Linear high signal was also visible, in FLAIR (Figure 1, panel C) and diffusion weighted imaging (Figure 1 , panel D), along the precentral and central sulci. Apparent diffusion coefficient mapping showed low signal in the mammillary bodies, consistent with water movement restriction, but normal values in the cortical lesions. With thiamine intravenous treatment, the patient gradually improved, being dismissed at day 9 with no ataxia or ocular signs but with a slight difficulty in retaining new information. An MRI taken at month 2 of follow-up showed only slight atrophy in the mammillary bodies.

\section{Discussion}

Linear cortical hyperintensities in T2-weighted imaging in a ribbonlike fashion were described in postictal stages, posterior reversible leucoencephalopathy, and postanoxic encephalopathy and, furthermost, sporadic Creutzfeldt-Jakob disease. ${ }^{3,4}$ Cortical damage in Wernicke's encephalopathy, in the rare cases it was reported, essentially affected the pericentral regions in a symmetric pattern. ${ }^{3,5-8}$ It was proposed that this feature would have clinical (responsible for the motor 
findings), ${ }^{3}$ as well as prognostic (indicating worst outcome), relevance. ${ }^{5}$ In our patient, this was not encountered as there was no motor defect and the patient had a good general outcome. The precocity of MRI assessment and thiamine reposition in our patient probably prevented us from seeing more extensive damage to the pericentral regions and these severe clinical consequences. Supporting this hypothesis is the absence of restriction to water molecules movement that we have observed: this finding is consistent with vasogenic edema, and as this is typically reversible, it can be a relevant prognostic feature, indicating that the metabolic damage is not permanent and it will be treatment-responsive. Furthermore, as the histological findings in Wernicke's encephalopathy confirm the existence of both cytotoxic and vasogenic edema, it is possible that one (the vasogenic) precedes the other (the cytotoxic), making diffusion weighted imaging an excellent tool to define the time window for successful thiamine treatment.

Previously presented as a poster at the annual meeting of the Portuguese Neurological Society, November 16, 2008.

Álvaro Machado, M.D. Neurology Department, Hospital de São Marcos, Braga, Portugal

MANUEl Ribeiro, M.D.

JoÃo SoARes-Fernandes, M.D. Neuroradiology Department, Hospital de São Marcos, Braga João Cerqueira, M.D., Ph.D.

Ricardo Maré, M.D.

Neurology Department, Hospital de São Marcos, Braga

\section{References}

1. Victor M, Adams RD, Collins GH: The Wernicke-Korsakoff syndrome: a clinical and pathological study of 245 patients, 82 with post-mortem examinations. Contemp Neurol Ser 1971; 7:1-206
2. Sechi G, Serra A: Wernicke's encephalopathy: new clinical settings and recent advances in diagnosis and management. Lancet Neurol 2007; 6:442-455

3. Zhong C, Jin L, Fei G: MR imaging of nonalcoholic Wernicke encephalopathy: a follow-up study. AJNR Am J Neuroradiol 2005; 26:2301-2305

4. Demaerel P, Sciot R, Robberecht W, et al: Accuracy of diffusion-weighted MR imaging in the diagnosis of sporadic Creutzfeldt-Jakob disease. J Neurol 2003; 250:222-225

5. Yamashita M, Yamamoto T: Wernicke encephalopathy with symmetric pericentral involvement: MR findings. J Comput Assist Tomogr 1995; 19:306-308

6. D'Aprile P, Tarantino A, Santoro N, et al: Wernicke's encephalopathy induced by total parenteral nutrition in patient with acute leukemia: unusual involvement of caudate nuclei and cerebral cortex on MRI. Neuroradiology 2000; 42:781-783

7. Doss A, Mahad D, Romanowski CA: Wernicke encephalopathy: unusual findings in nonalcoholic patients. J Comput Assist Tomogr 2003; 27:235-240

8. Liu YT, Fuh JL, Lirng JF, et al: Correlation of magnetic resonance images with neuropathology in acute Wernicke's encephalopathy. Clin Neurol Neurosurg 2006; 108:682-687 